\title{
Adaptive Synchronization of Complex Dynamical Multilinks Networks with Similar Nodes
}

\author{
Weiping Wang, ${ }^{1}$ Lixiang Li, ${ }^{2}$ Haipeng Peng, ${ }^{2}$ Jialiang Yuan, ${ }^{2}$ \\ Jinghua Xiao, ${ }^{1}$ and Yixian Yang ${ }^{2,3}$ \\ ${ }^{1}$ School of Science, Beijing University of Posts and Telecommunications, Beijing 100876, China \\ ${ }^{2}$ Information Security Center, Beijing University of Posts and Telecommunications, Beijing 100876, China \\ ${ }^{3}$ National Engineering Laboratory for Disaster Backup and Recovery, Beijing University of Posts and Telecommunications, \\ Beijing 100876, China
}

Correspondence should be addressed to Lixiang Li; li_lixiang2006@163.com

Received 2 May 2013; Accepted 21 May 2013

Academic Editor: Ming Li

Copyright (C) 2013 Weiping Wang et al. This is an open access article distributed under the Creative Commons Attribution License, which permits unrestricted use, distribution, and reproduction in any medium, provided the original work is properly cited.

\begin{abstract}
This paper studies the synchronization of complex dynamical networks with multilinks and similar nodes. The dynamics of all the nodes in the networks are impossible to be completely identical due to the differences of parameters or the existence of perturbations. Networks with similar nodes are universal in the real world. In order to depict the similarity of the similar nodes, we give the definition of the minimal similarity of the nodes in the network for the first time. We find the threshold of the minimal similarity of the nodes in the network. If the minimal similarity of the nodes is bigger than the threshold, then the similar nodes can achieve synchronization without controllers. Otherwise, adaptive synchronization method is adopted to synchronize similar nodes in the network. Some new synchronization criteria are proposed based on the Lyapunov stability theory. Finally, numerical simulations are given to illustrate the feasibility and the effectiveness of the proposed theoretical results.
\end{abstract}

\section{Introduction}

Complex dynamical networks have attracted increasing attention in recent years, since they have been widely exploited to model many complex systems in the science, engineering, and society $[1,2]$. Synchronization of complex network has been found to be a universal phenomenon in nature and it has important potential applications to realword dynamical systems. As an important and interesting collective behavior, synchronization of complex network has been studied extensively [3-8], such as complete synchronization, projective synchronization [9], impulsive synchronization [10, 11], exponential synchronization [12], adaptive synchronization [13-15], and pinning synchronization [1621].

Most previous research assumes that the dynamics of all nodes are identical. Consequently, the synchronization problem is significantly simplified. However, the assumption that the nodes are completely identical is not realistic in many real-world networks [22], such as in the neural networks, where the internal neurons in the nervous system are impossible to be completely identical due to the differences of the parameters. And the authors of $[23,24]$ studied synchronization of complex dynamical networks with nonidentical nodes. While, in normal circumstances, the neurons are not completely identical or completely nonidentical. They are similar to each other and they will achieve synchronization to transmit information which shows that the neural system has certain robustness. At this time, we want to know the answers of the following questions, which have a practical meaning for us to analyze and control many realistic networks with similar nodes. How to depict the similarity of the similar nodes? What is the condition that the similar nodes have to satisfy in the network in order to achieve synchronization without controllers? If there is a mutation or a pathological change, then some neurons may have many different characteristics, 
and they can not synchronize with other neurons. When the similarity of the similar nodes is broken, how to synchronize the nodes in the network?

Furthermore, enormous works have been done on the synchronization in complex networks with single-link, and a lot of meaningful conclusions have been obtained. The authors of [19] propose that single-link network is a special case of multilinks network. Therefore, research on multilinks networks are more representative. Multilinks means that there is more than one link between two nodes and each of them has its own property. For instance, there are relationship networks, transportation networks, World Wide Web, and so forth. The transportation network as an example of a network with multilinks, which is made up by combining the corresponding airline network, railway network, and highway network. We can split the multilinks networks into many subnetworks based on the property of the connections. For a transportation network, the transmission speed is different among airline network, railway network, and highway network. In our previous work [19], time-delay was introduced to split complex dynamical networks into subnetworks, upon which a model of complex networks with multilinks has been constructed. However, the important issue of synchronization for complex dynamical networks with similar nodes and multilinks has so far received little attention. The study of the synchronization problem with similar nodes in the complex multilinks network becomes an interesting and challenging topic.

In this paper, we give a model of complex multilinks networks with similar nodes. A definition of similar nodes is given and the minimal similarity of similar nodes in the network is analyzed for the first time. We find a threshold of the minimal similarity of similar nodes. If the minimal similarity of similar nodes is bigger than the threshold in the network, then the similar nodes can achieve synchronization. Otherwise, we should add some controllers to the nodes in order to get synchronization. Then some new adaptive synchronization criteria are proposed. Finally, numerical simulations of dynamical networks with similar nodes are presented to demonstrate the feasibility and the effectiveness of the results.

\section{Model and Preliminaries}

The model of complex multilinks network consisting of $N$ similar nodes with $m$ kinds of properties can be described by

$$
\begin{aligned}
\dot{x}_{i}(t)= & A_{i} x_{i}(t)+f\left(x_{i}(t)\right)+c \sum_{j=1}^{N} b_{(0) i j} \Gamma_{0} x_{j}(t) \\
& +c \sum_{l=1}^{m-1} \sum_{j=1}^{N} b_{(l) i j} \Gamma_{l} x_{j}\left(t-\tau_{l}\right), \quad i=1,2, \ldots, N,
\end{aligned}
$$

where $x_{i}(t)=\left(x_{i 1}(t), x_{i 2}(t), \ldots, x_{i N}(t)\right)^{T}$ is the state vector of node $i, A_{i}$ is a matrix, $f\left(x_{i}(t)\right)$ is a smooth nonlinear vector function, $c>0$ is the coupling strength, $\Gamma_{0}$ and $\Gamma_{l}$ are the inner-coupling matrices, $B_{l}=\left(b_{(l) i j}\right)_{N \times N}, 0 \leq l \leq m-1$ represents the topological structure of the $l$ th subnetwork, and $\tau_{l}$ is the time-delay of the $l$ th subnetwork compared with the basic network $\left(\tau_{0}=0\right)$. We define $b_{(l) i j}=b_{(l) j i}=1$ if there is a connection between node $i$ and node $j(j \neq i)$ in the $l$ th subnetwork, otherwise $b_{(l) i j}=b_{(l) j i}=0$. And we define $b_{(l) i i}=-\sum_{j=1, j \neq i}^{N} b_{(l) i j}$.

Network (1) is in a state of asymptotical synchronization, if

$$
x_{1}(t)=x_{2}(t)=\cdots=x_{N}(t) \longrightarrow s(t)
$$

as $t \rightarrow \infty(1 \leq i \leq N)$, where $s(t) \epsilon R^{n}$ is a synchronous solution of the node system $\dot{x}_{i}(t)=A_{i} x_{i}(t)+f\left(x_{i}(t)\right)$. We define the error vectors as

$$
e_{i}(t)=x_{i}(t)-s_{i}(t) .
$$

Hereafter, the definitions of similar nodes and the minimal similarity of the similar nodes are given, and a useful assumption and two lemmas are introduced.

Definition 1. If $A_{i}$ and $A_{j}$ are matrices with the similar element values, then the node $i$ and $j$ are similar nodes. In the network (1), we define $A$ as the matrix of basic node and $A_{i}(1 \leq i \leq N-1)$ as matrices of other nodes. Because $A$ and $A_{i}$ are matrices with similar element values, we define $\delta_{i}=1-\left(\left\|A-A_{i}\right\|_{F} /\|A\|_{F}\right), v=\min \left(\delta_{i}\right),(1 \leq i \leq N-1)$, and $\nu(0<v<1)$ represents the minimal similarity of the nodes in the network. The norm of matrix $A$ is $\|A\|_{F}=\left(\sum_{i, j=1}^{N} a_{i j}^{2}\right)^{1 / 2}$, and $a_{i j}(1 \leq i, j \leq N)$ are elements of matrix $A$.

Remark 2. From Definition 1, we know $v$ is an important parameter. When $v$ approaches to 1 , the nodes in the network are similar. If $v$ satisfies a certain condition, then the similar nodes of the network can achieve synchronization without controllers. On the contrary, when $v$ is far away from 1 , the nodes in the network become not similar, so the nodes cannot achieve synchronization without controllers. That is to say, there exists a threshold, if $v$ is bigger than the threshold; then the similar nodes in the network can get synchronization without controllers. And the threshold is what we tried to find in the following.

Assumption 3. The smooth nonlinear function $f(\cdot)$ satisfies the following Lipschitz condition:

$$
\left\|f\left(x_{i}(t)\right)-f(s(t))\right\| \leq L\left\|x_{i}(t)-s(t)\right\|,
$$

where $L$ is a positive constant.

Lemma 4. For any two vectors $x$ and $y$, and a matrix $Q>0$ with compatible dimensions, one has

$$
2 x^{T} y \leq x^{T} Q x+y^{T} Q^{-1} y .
$$

Lemma 5. If $A \epsilon C^{N \times N}$, the eigenvalues of $A$ are $\lambda_{i}(i=$ $1,2, \ldots, N)$, then $\max \left(\lambda_{i}\right) \leq\|A\|$, where $\|A\|$ is an arbitrary matrix norm.

\section{Synchronization Analysis}

In this section, suppose there is not a control scheme to synchronize a delayed complex multilinks network with 
similar nodes. According to system (1), the error dynamical system can be derived as

$$
\begin{aligned}
\dot{e}_{i}(t)= & A_{i} e_{i}(t)+f\left(x_{i}(t)\right)-f(s(t))+c \sum_{j=1}^{N} b_{(0) i j} \Gamma_{0} e_{j}(t) \\
& +c \sum_{l=1}^{m-1} \sum_{j=1}^{N} b_{(l) i j} \Gamma_{l} e_{j}\left(t-\tau_{l}\right),
\end{aligned}
$$

where $A_{i}=A+\Delta A_{i}, A_{i}$ is the matrix of node $i$, and $A$ is the matrix of basic node. Because $A$ and $A_{i}$ are matrices with similar element values, $\Delta A_{i}=A_{i}-A$. It is easy to see that the synchronization of the complex network (1) is achieved if the zero solution of the error system (6) is globally asymptotically stable, which is ensured by the following theorem. And we find that the minimal similarity of the similar nodes satisfies an inequality for synchronization.

Theorem 6. Consider network (1), if the minimal similarity of the nodes $v$ is bigger than the threshold, where the threshold of $v$ is

$$
\begin{aligned}
& \left(\lambda_{\max }(A)+L+c \lambda_{\max }\left(P_{0}\right)+\frac{c}{2} \sum_{l=1}^{l=m-1} \lambda_{\max }\left(P_{l} P_{l}^{T}\right)\right. \\
& \left.+\frac{c}{2}(m-1)+\|A\|_{F}\right)\left(\|A\|_{F}\right)^{-1},
\end{aligned}
$$

and it also satisfies the following inequality:

$$
\begin{gathered}
\frac{\lambda_{\text {max }}(A)+L+c \lambda_{\text {max }}\left(P_{0}\right)+(c / 2) \sum_{l=1}^{l=m-1} \lambda_{\max }\left(P_{l} P_{l}^{T}\right)}{\|A\|_{F}} \\
+\frac{(c / 2)(m-1)+\|A\|_{F}}{\|A\|_{F}}<v<1,
\end{gathered}
$$

then the system (1) is synchronized without controllers.

Proof. Construct the following Lyapunov function:

$$
V(t)=\frac{1}{2} \sum_{i=1}^{N} e_{i}(t)^{T} e_{i}(t)+\frac{c}{2} \sum_{l=1}^{m-1} \int_{t-\tau_{l}}^{t} \sum_{i=1}^{N} e_{i}(\theta)^{T} e_{i}(\theta) d \theta .
$$

We get

$$
\begin{aligned}
\dot{V}(t)= & \sum_{i=1}^{N} e_{i}(t)^{T} \dot{e}_{i}(t) \\
& +\frac{c}{2} \sum_{l=1}^{m-1} \sum_{i=1}^{N}\left[e_{i}(t)^{T} e_{i}(t)-e_{i}\left(t-\tau_{l}\right)^{T} e_{i}\left(t-\tau_{l}\right)\right] \\
= & \sum_{i=1}^{N} e_{i}(t)^{T} A e_{i}(t)+\sum_{i=1}^{N} e_{i}(t)^{T} \Delta A_{i} e_{i}(t) \\
& +\sum_{i=1}^{N} e_{i}(t)\left[f\left(x_{i}(t)\right)-f(s(t))\right]
\end{aligned}
$$

$$
\begin{aligned}
& +\sum_{i=1}^{N} c e_{i}(t)^{T} \sum_{j=1}^{N} b_{(0) i j} \Gamma_{0} e_{j}(t) \\
& +\sum_{i=1}^{N} c e_{i}(t)^{T} \sum_{l=1}^{m-1} \sum_{j=1}^{N} b_{(l) i j} \Gamma_{l} e_{j}\left(t-\tau_{l}\right) \\
& +\frac{c}{2} \sum_{i=1}^{N} \sum_{l=1}^{m-1} e_{i}(t)^{T} e_{i}(t) \\
& -\frac{c}{2} \sum_{i=1}^{N} \sum_{l=1}^{m-1} e_{i}\left(t-\tau_{l}\right)^{T} e_{i}\left(t-\tau_{l}\right) .
\end{aligned}
$$

$$
\begin{aligned}
\text { Let } e(t) & =\left(e_{1}(t), e_{2}(t), \ldots, e_{N}(t)\right), \text { then we get } \\
\dot{V}(t) \leq & {\left[e(t)^{T} A e(t)+e(t)^{T} \Delta A_{i} e(t)+L e(t)^{T} e(t)\right] } \\
& +c e(t)^{T}\left(B_{0} \otimes \Gamma_{0}\right) e(t) \\
& +c e(t)^{T}\left(B_{1} \otimes \Gamma_{1}\right) e\left(t-\tau_{1}\right) \\
& +c e(t)^{T}\left(B_{2} \otimes \Gamma_{2}\right) e\left(t-\tau_{2}\right) \\
& +\cdots+c e(t)^{T}\left(B_{m-1} \otimes \Gamma_{m-1}\right) e\left(t-\tau_{m-1}\right) \\
& +\frac{c}{2}(m-1) e(t)^{T} e(t) \\
& -\frac{c}{2} e\left(t-\tau_{1}\right)^{T} e\left(t-\tau_{1}\right)-\frac{c}{2} e\left(t-\tau_{2}\right)^{T} e\left(t-\tau_{2}\right) \\
& -\cdots-\frac{c}{2} e\left(t-\tau_{m-1}\right)^{T} e\left(t-\tau_{m-1}\right) .
\end{aligned}
$$

Let

$$
\begin{aligned}
P_{0} & =B_{0} \otimes \Gamma_{0}, \\
P_{1} & =B_{1} \otimes \Gamma_{1}, \\
P_{2} & =B_{1} \otimes \Gamma_{2}, \\
& \vdots \\
P_{m-1} & =B_{m-1} \otimes \Gamma_{m-1},
\end{aligned}
$$

where $\otimes$ represents the Kronecker product. Then by Lemma 4, we have

$$
\begin{aligned}
\dot{V}(t) \leq & \lambda_{\max }(A) e(t)^{T} e(t)+\lambda_{\max }\left(\Delta A_{i}\right) e(t)^{T} e(t) \\
& +L e(t)^{T} e(t)+c \lambda_{\max }\left(P_{0}\right) e(t)^{T} e(t) \\
& +\frac{c}{2} \lambda_{\max }\left(P_{1} P_{1}^{T}\right) e(t)^{T} e(t) \\
& +\frac{c}{2} \lambda_{\max }\left(P_{2} P_{2}^{T}\right) e(t)^{T} e(t) \\
& +\cdots+\frac{c}{2} \lambda_{\max }\left(P_{m-1} P_{m-1}^{T}\right) e(t)^{T} e(t)
\end{aligned}
$$




$$
\begin{aligned}
& +\frac{c}{2}(m-1) e(t)^{T} e(t) \\
\leq & {\left[\lambda_{\max }(A)+\left\|\Delta A_{i}\right\|_{F}+L+c \lambda_{\max }\left(P_{0}\right)\right.} \\
& \left.+\frac{c}{2} \sum_{l=1}^{m-1} \lambda_{\max }\left(P_{l} P_{l}^{T}\right)+\frac{c}{2}(m-1)\right] e(t)^{T} e(t) \\
\leq & {\left[\begin{array}{l}
\lambda_{\max }(A)+(1-v)\|A\|_{F}+L+c \lambda_{\max }\left(P_{0}\right) \\
+
\end{array}\right.} \\
& \left.\frac{c}{2} \sum_{l=1}^{m-1} \lambda_{\max }\left(P_{l} P_{l}^{T}\right)+\frac{c}{2}(m-1)\right] e(t)^{T} e(t) .
\end{aligned}
$$

Therefore, if we have

$$
\begin{array}{r}
\lambda_{\max }(A)+(1-v)\|A\|_{F}+L+c \lambda_{\max }\left(P_{0}\right) \\
+\frac{c}{2} \sum_{l=1}^{m-1} \lambda_{\max }\left(P_{l} P_{l}^{T}\right)+\frac{c}{2}(m-1)<0
\end{array}
$$

then $\dot{V}(t) \leq 0$. So we get the synchronization criterion as follows:

$$
\begin{gathered}
\frac{\lambda_{\text {max }}(A)+L+c \lambda_{\max }\left(P_{0}\right)+(c / 2) \sum_{l=1}^{l=m-1} \lambda_{\max }\left(P_{l} P_{l}^{T}\right)}{\|A\|_{F}} \\
+\frac{(c / 2)(m-1)+\|A\|_{F}}{\|A\|_{F}}<v<1 .
\end{gathered}
$$

If $v$ satisfies (15), the nodes are synchronized. Thus we complete the proof.

Remark 7. The matrix of basic node can be chosen at random from $A_{i}(1 \leq i \leq N)$. No matter which one we choose, Theorem 6 also holds.

Furthermore, noise plays an important role in the process of synchronization. Here we consider the influence of the noise. If there is an additive noise in the system (1) in the form of

$$
\begin{gathered}
\dot{x}_{i}(t)=A_{i} x_{i}(t)+f\left(x_{i}(t)\right)+c \sum_{j=1}^{N} b_{(0) i j} \Gamma_{0} x_{j}(t) \\
+c \sum_{l=1}^{m-1} \sum_{j=1}^{N} b_{(l) i j} \Gamma_{l} x_{j}\left(t-\tau_{l}\right)+\eta_{i}(t), \\
i=1,2, \ldots, N,
\end{gathered}
$$

where $\eta_{i}(t) \epsilon R^{n}$ is the zero mean bounded noise. Using system (16), we can easily get the following error system:

$$
\begin{aligned}
\dot{e}_{i}(t)= & \left(A+\Delta A_{i}\right) e_{i}(t)+f\left(x_{i}(t)\right)-f(s(t)) \\
& +c \sum_{j=1}^{N} b_{(0) i j} \Gamma_{0} e_{j}(t) \\
& +c \sum_{l=1}^{m-1} \sum_{j=1}^{N} b_{(l) i j} \Gamma_{l} e_{j}\left(t-\tau_{l}\right)+\eta_{i}(t)
\end{aligned}
$$

then we get

$$
\begin{aligned}
E\left(\dot{e}_{i}(t)\right)= & \left(A+\Delta A_{i}\right) E\left[e_{i}(t)\right]+E\left[f\left(x_{i}(t)\right)-f(s(t))\right] \\
& +c \sum_{j=1}^{N} b_{(0) i j} \Gamma_{0} E\left[e_{j}(t)\right] \\
& +c \sum_{l=1}^{m-1} \sum_{j=1}^{N} b_{(l) i j} \Gamma_{l} E\left[e_{j}\left(t-\tau_{l}\right)\right]+E\left[\eta_{i}(t)\right] .
\end{aligned}
$$

Finally, we get the theorem as follows.

Theorem 8. When there is a noise or perturbation, considering the network (16), if the following condition holds

$$
\begin{gathered}
\frac{\lambda_{\text {max }}(A)+L+c \lambda_{\text {max }}\left(P_{0}\right)+(c / 2) \sum_{l=1}^{l=m-1} \lambda_{\text {max }}\left(P_{l} P_{l}^{T}\right)}{\|A\|_{F}} \\
+\frac{(c / 2)(m-1)+\|A\|_{F}}{\|A\|_{F}}<v<1,
\end{gathered}
$$

then $E\left[e_{i}(t)\right]$ approaches to zero.

The proof process of Theorem 8 is similar to the proof process of Theorem 6 , so here it is omitted.

\section{Adaptive Synchronization}

In this section, a control scheme is developed to synchronize a delayed complex multilinks network with similar nodes, which do not satisfy the synchronization criterion (15). And the following adaptive controllers are used:

$$
u_{i}=-d_{i} e_{i}(t), \quad 1 \leq i \leq N
$$

And the updating laws are

$$
\dot{d}_{i}=k_{i} e_{i}(t)^{T} e_{i}(t), \quad 1 \leq i \leq N
$$

where $k_{i}(1 \leq i \leq N)$ are positive constants. The adaptive controllers (20) are widely used in solving many synchronous problems. 
Then the controlled network can be characterized as

$$
\begin{gathered}
\dot{x}_{i}(t)=A_{i} x_{i}(t)+f\left(x_{i}(t)\right)+c \sum_{j=1}^{N} b_{(0) i j} \Gamma_{0} x_{j}(t) \\
+c \sum_{l=1}^{m-1} \sum_{j=1}^{N} b_{(l) i j} \Gamma_{l} x_{j}\left(t-\tau_{l}\right)+u_{i}, \\
i=1,2, \ldots, N .
\end{gathered}
$$

According to system (22), the following error dynamical system can be derived:

$$
\begin{aligned}
\dot{e}_{i}(t)= & \left(A+\Delta A_{i}\right) e_{i}(t)+f\left(x_{i}(t)\right)-f(s(t)) \\
& +c \sum_{j=1}^{N} b_{(0) i j} \Gamma_{0} e_{j(t)} \\
& +c \sum_{l=1}^{m-1} \sum_{j=1}^{N} b_{(l) i j} \Gamma_{l} e_{j}\left(t-\tau_{l}\right)-d_{i} e_{i}(t) .
\end{aligned}
$$

It is clear to see that the synchronization of the controlled complex network (22) is achieved if the zero solution of the error system (23) is globally asymptotically stable, which is ensured by the following theorem.

Theorem 9. Consider the network (22) under the actions of the controllers (20) and the updating laws (21). If the following condition holds:

$$
\begin{gathered}
\lambda_{\max }(A)+(1-v)\|A\|_{F}+L+c \lambda_{\max }\left(P_{0}\right) \\
+\frac{c}{2} \sum_{l=1}^{l=m-1} \lambda_{\max }\left(P_{l} P_{l}^{T}\right)+\frac{c}{2}(m-1)<d^{*},
\end{gathered}
$$

where $d^{*}$ is a sufficiently large positive constant to be determined, then the system (1) is synchronized.

Proof. Construct the following Lyapunov function:

$$
\begin{aligned}
V(t)= & \frac{1}{2} \sum_{i=1}^{N} e_{i}(t)^{T} e_{i}(t) \\
& +\frac{c}{2} \sum_{l=1}^{m-1} \int_{t-\tau_{l}}^{t} \sum_{i=1}^{N} e_{i}(\theta)^{T} e_{i}(\theta) d \theta \\
& +\frac{1}{2} \sum_{i=1}^{N} \frac{\left(d_{i}-d^{*}\right)^{2}}{k_{i}} .
\end{aligned}
$$

Clearly, $V(t)$ is positive. Then the derivative of $V(t)$ is obtained as

$$
\begin{aligned}
& \dot{V}(t)=\sum_{i=1}^{N} e_{i}(t)^{T} \dot{e}_{i}(t) \\
& +\frac{c}{2} \sum_{l=1}^{m-1} \sum_{i=1}^{N}\left[e_{i}(t)^{T} e_{i}(t)\right. \\
& \left.-e_{i}\left(t-\tau_{l}\right)^{T} e_{i}\left(t-\tau_{l}\right)\right] \\
& +\sum_{i=1}^{N}\left[d_{i} e_{i}(t)^{T} e_{i}(t)-d^{*} e_{i}(t)^{T} e_{i}(t)\right] \\
& =\sum_{i=1}^{N}\left\{e_{i}(t)^{T} A e_{i}(t)+e_{i}(t)^{T} \Delta A_{i} e_{i}(t)+e_{i}(t)\right. \\
& \times\left[f\left(x_{i}(t)\right)-f(s(t))\right] \\
& +c e_{i}(t)^{T} \sum_{j=1}^{N} b_{(0) i j} \Gamma_{0} e_{j}(t) \\
& +c e_{i}(t)^{T} \sum_{l=1}^{m-1} \sum_{j=1}^{N} b_{(l) i j} \Gamma_{l} e_{j}\left(t-\tau_{l}\right) \\
& -d_{i} e_{i}(t)^{T} e_{i}(t)+\frac{c}{2} \sum_{l=1}^{m-1} e_{i}(t)^{T} e_{i}(t) \\
& -\frac{c}{2} \sum_{l=1}^{m-1} e_{i}\left(t-\tau_{l}\right)^{T} e_{i}\left(t-\tau_{l}\right) \\
& \left.+d_{i} e_{i}(t)^{T} e_{i}(t)-d^{*} e_{i}(t)^{T} e_{i}(t)\right\} .
\end{aligned}
$$

Let $e(t)=\left(e_{1}(t), e_{2}(t), \ldots, e_{N}(t)\right)$, then we get

$$
\begin{aligned}
\dot{V}(t) \leq & {\left[e(t)^{T} A e(t)+e(t)^{T} \Delta A_{i} e(t)+L e(t)^{T} e(t)\right] } \\
& +c e(t)^{T}\left(B_{0} \otimes \Gamma_{0}\right) e(t) \\
& +c e(t)^{T}\left(B_{1} \otimes \Gamma_{1}\right) e\left(t-\tau_{1}\right)+c e(t)^{T} \\
& \times\left(B_{2} \otimes \Gamma_{2}\right) e\left(t-\tau_{2}\right) \\
& +\cdots+c e(t)^{T}\left(B_{m-1} \otimes \Gamma_{m-1}\right) e\left(t-\tau_{m-1}\right) \\
& +\frac{c}{2}(m-1) e(t)^{T} e(t)-\frac{c}{2} e\left(t-\tau_{1}\right)^{T} e\left(t-\tau_{1}\right) \\
& -\frac{c}{2} e\left(t-\tau_{2}\right)^{T} e\left(t-\tau_{2}\right) \\
& -\cdots-\frac{c}{2} e\left(t-\tau_{m-1}\right)^{T} e\left(t-\tau_{m-1}\right)-d^{*} e(t)^{T} e(t) .
\end{aligned}
$$




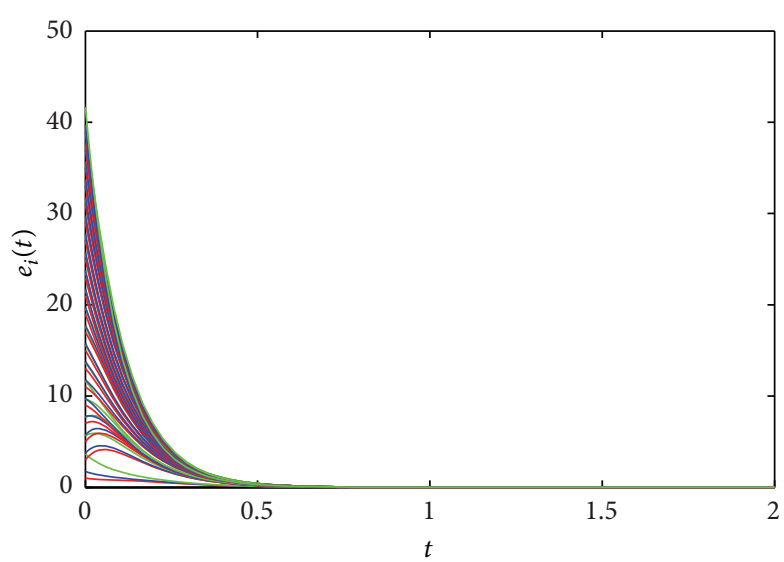

(a)

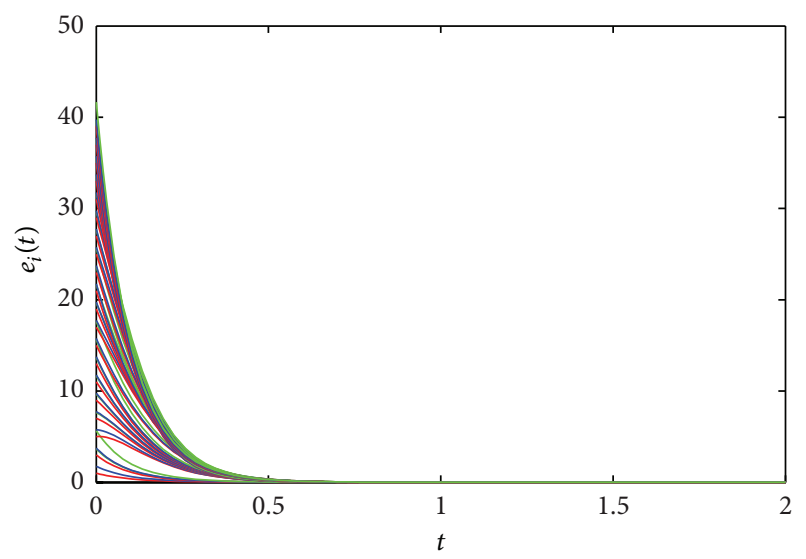

(c)

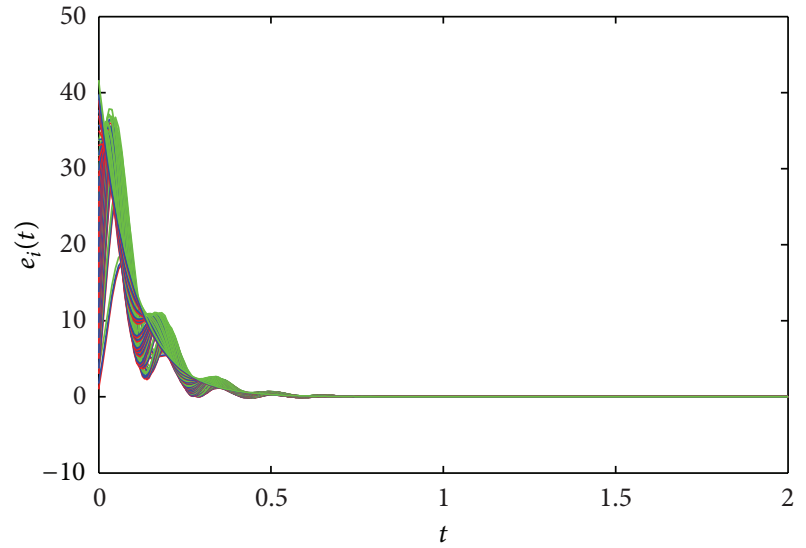

(b)

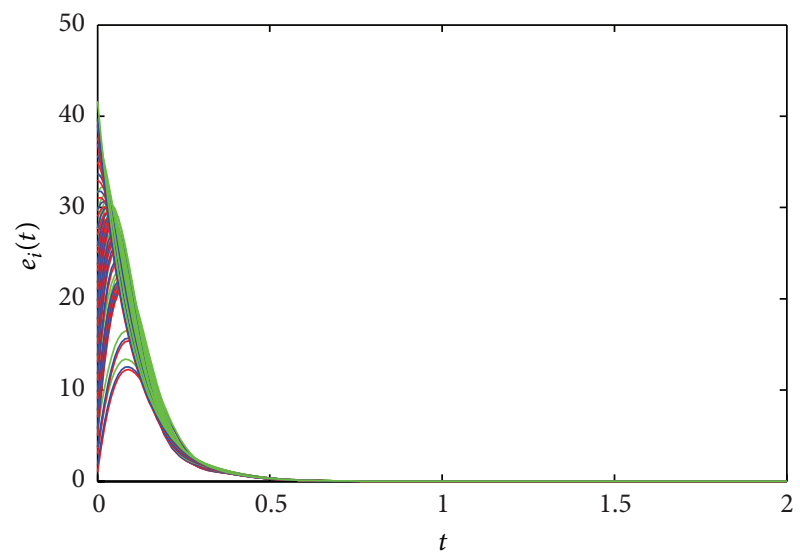

(d)

FIGURE 1: Separate synchronous variables $e_{i 1}, e_{i 2}, e_{i 3}(1 \leqslant i \leqslant 30)$ of different network models where $e_{i 1}$ are shown by the red line, $e_{i 2}$ are shown by the blue line, and $e_{i 3}$ are shown by the green line. $c=2 ; \tau=0.01$. (a) $B_{0}$ and $B_{1}$ are small-world network models, and the rewiring probability among nodes is 0.3 and 0.6. (b) $B_{0}$ and $B_{1}$ are scale-free network models, and their minimum degrees are 2 and 3 . (c) $B_{0}$ and $B_{1}$ are random network models, and their connection probability among nodes is 0.1 and 0.3 . (d) $B_{0}$ is a small-world network model, and the rewiring probability among nodes is $0.3 . B_{1}$ is a scale-free network model, and the minimum degree is 2 . Those similar nodes can achieve synchronization without controllers (color online).

Let

$$
\begin{gathered}
P_{0}=B_{0} \otimes \Gamma_{0}, \\
P_{1}=B_{1} \otimes \Gamma_{1}, \\
P_{2}=B_{1} \otimes \Gamma_{2}, \\
\vdots \\
P_{m-1}=B_{m-1} \otimes \Gamma_{m-1},
\end{gathered}
$$

where $\otimes$ represents the Kronecker product. Then by Lemma 4, we have

$$
\begin{aligned}
\dot{V}(t) \leq & \lambda_{\max }(A) e(t)^{T} e(t) \\
& +\lambda_{\max }\left(\Delta A_{i}\right) e(t)^{T} e(t)+L e(t)^{T} e(t) \\
& +c \lambda_{\max }\left(P_{0}\right) e(t)^{T} e(t) \\
& +\frac{c}{2} \lambda_{\max }\left(P_{1} P_{1}^{T}\right) e(t)^{T} e(t) \\
& +\cdots+\frac{c}{2} \lambda_{\max }\left(P_{m-1} P_{m-1}^{T}\right) e(t)^{T} e(t)
\end{aligned}
$$

$$
\begin{aligned}
& +\frac{c}{2}(m-1) e(t)^{T} e(t)-d^{*} e(t)^{T} e(t) \\
& \leq\left[\lambda_{\max }(A)+\left\|\Delta A_{i}\right\|_{F}+L\right. \\
& +c \lambda_{\max }\left(P_{0}\right)+\frac{c}{2} \sum_{l=1}^{m-1} \lambda_{\max }\left(P_{l} P_{l}^{T}\right) \\
& \left.+\frac{c}{2}(m-1)-d^{*}\right] e(t)^{T} e(t) \\
& \leq\left[\lambda_{\max }(A)+(1-v)\|A\|_{F}+L\right. \\
& +c \lambda_{\max }\left(P_{0}\right)+\frac{c}{2} \sum_{l=1}^{m-1} \lambda_{\max }\left(P_{l} P_{l}^{T}\right) \\
& \left.+\frac{c}{2}(m-1)-d^{*}\right] e(t)^{T} e(t) .
\end{aligned}
$$




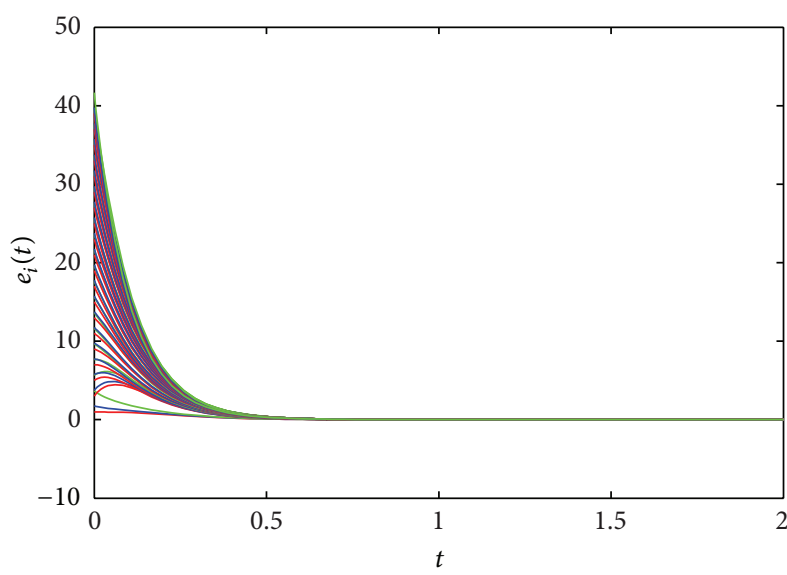

(a)

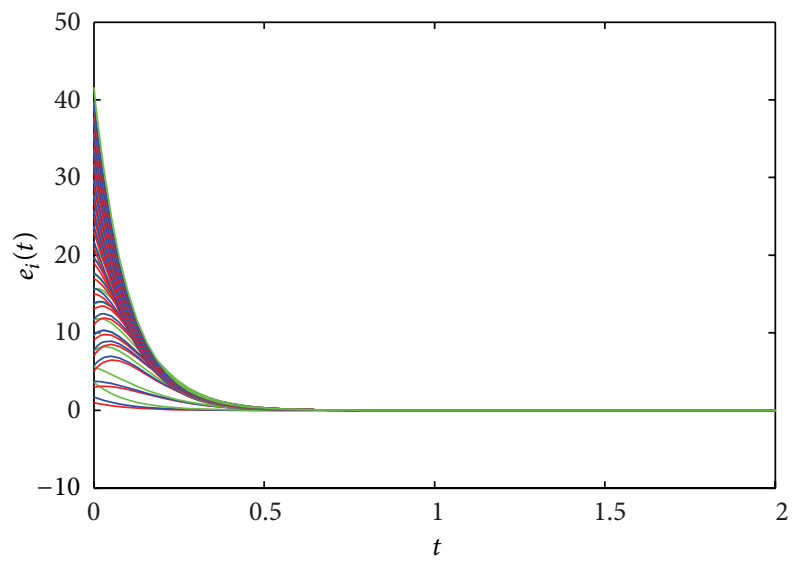

(c)

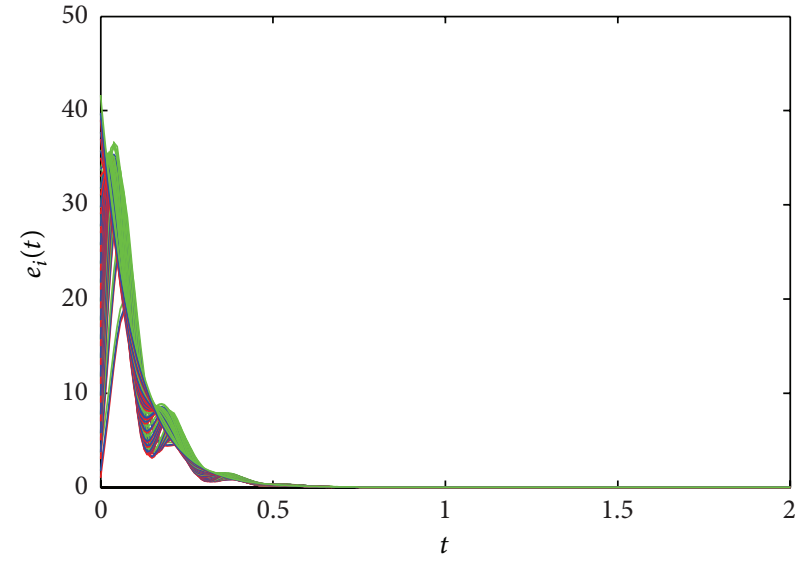

(b)

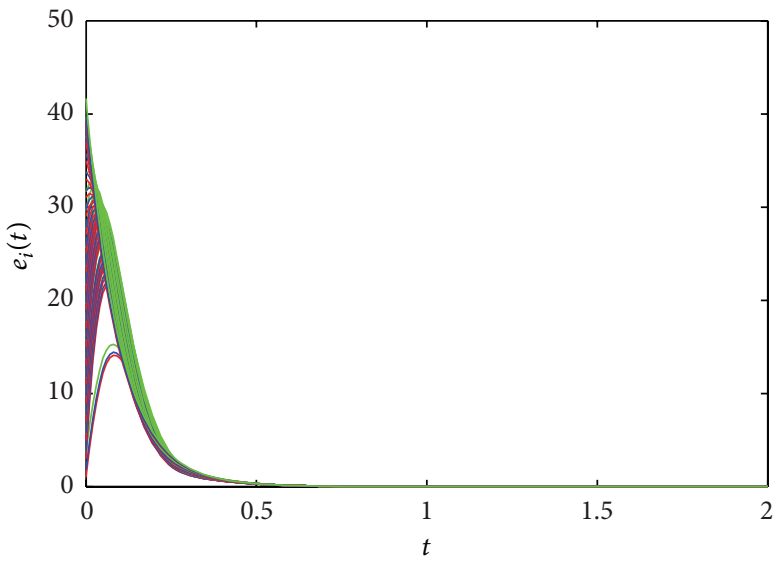

(d)

Figure 2: The similar nodes can achieve synchronization without controllers when the Brownian motion satisfies $E \omega(t)=0, D \omega(t)=1$. And separate synchronous variables $e_{i 1}, e_{i 2}, e_{i 3}(1 \leqslant i \leqslant 30)$ of different network models where $e_{i 1}$ are shown by the red line, $e_{i 2}$ are shown by the blue line, and $e_{i 3}$ are shown by the green line. $c=2 ; \tau=0.01$. The network models of (a) $-(\mathrm{d})$ are the same with Figures $1(\mathrm{a})-1(\mathrm{~d})$ (color online).

Therefore, if we have

$$
\begin{array}{r}
\lambda_{\max }(A)+(1-v)\|A\|_{F}+L+c \lambda_{\max }\left(P_{0}\right) \\
+\frac{c}{2} \sum_{l=1}^{m-1} \lambda_{\max }\left(P_{l} P_{l}^{T}\right)+\frac{c}{2}(m-1)<d^{*},
\end{array}
$$

then $\dot{V}(t) \leq 0$. Here we complete the proof.

Remark 10. If there is not a nonlinear function in system (1), then the network (1) is transferred into

$$
\begin{array}{r}
\dot{x}_{i}(t)=A_{i} x_{i}(t)+c \sum_{j=1}^{N} b_{(0) i j} \Gamma_{0} x_{j(t)} \\
+c \sum_{l=1}^{m-1} \sum_{j=1}^{N} b_{(l) i j} \Gamma_{l} x_{j}\left(t-\tau_{l}\right), \\
i=1,2, \ldots, N .
\end{array}
$$

Likewise, we can design the controllers as in (20) and (21). If the following condition holds:

$$
\begin{aligned}
\lambda_{\max }(A)+(1-\nu)\|A\|_{F}+c \lambda_{\max }\left(P_{0}\right) & \\
& +\frac{c}{2} \sum_{l=1}^{m-1} \lambda_{\max }\left(P_{l} P_{l}^{T}\right)+\frac{c}{2}(m-1)<d^{*}
\end{aligned}
$$

then the system (31) is synchronized, where $d^{*}$ is a sufficiently large positive constant to be determined.

Remark 11. The single-link network is a special case of multilinks networks [19]. When there is not a delay, the network (1) is transferred into the following single-link network:

$$
\begin{aligned}
\dot{x}_{i}(t)= & A_{i} x_{i}(t)+f\left(x_{i}(t)\right) \\
& +c \sum_{j=1}^{N} b_{(0) i j} \Gamma_{0} x_{j}(t), \quad i=1,2, \ldots, N,
\end{aligned}
$$




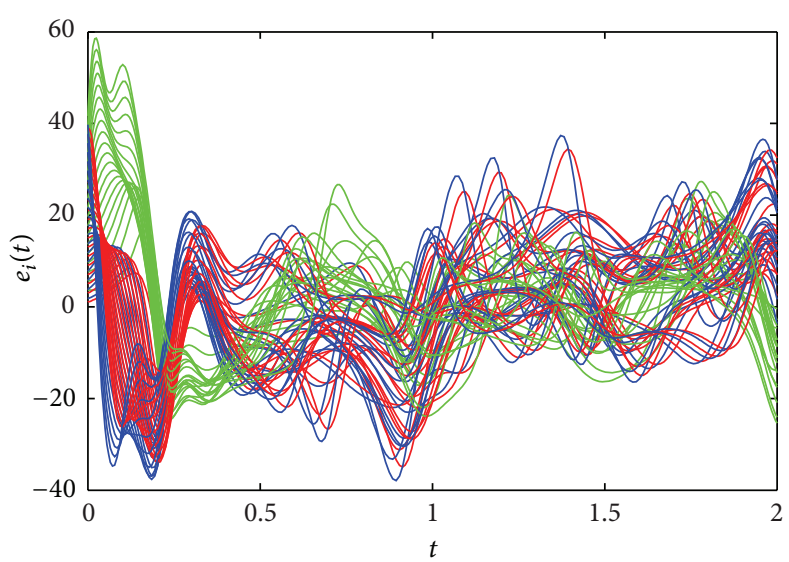

(a)

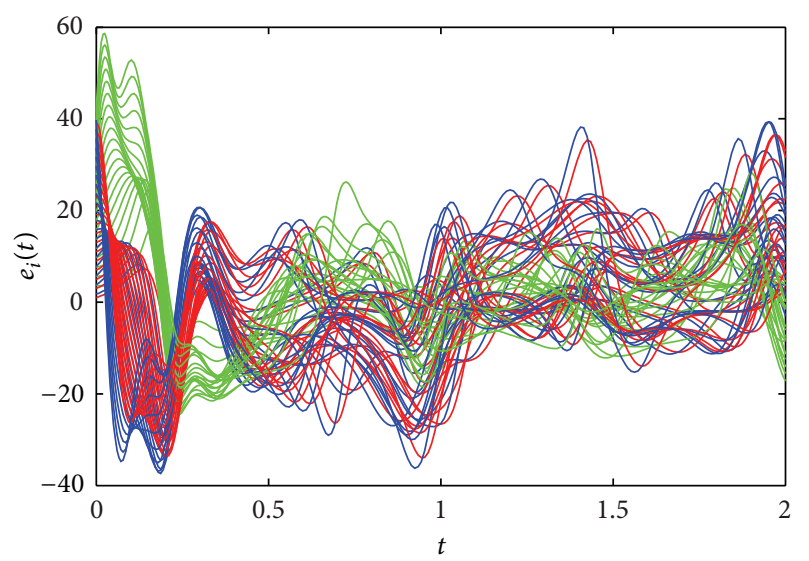

(c)

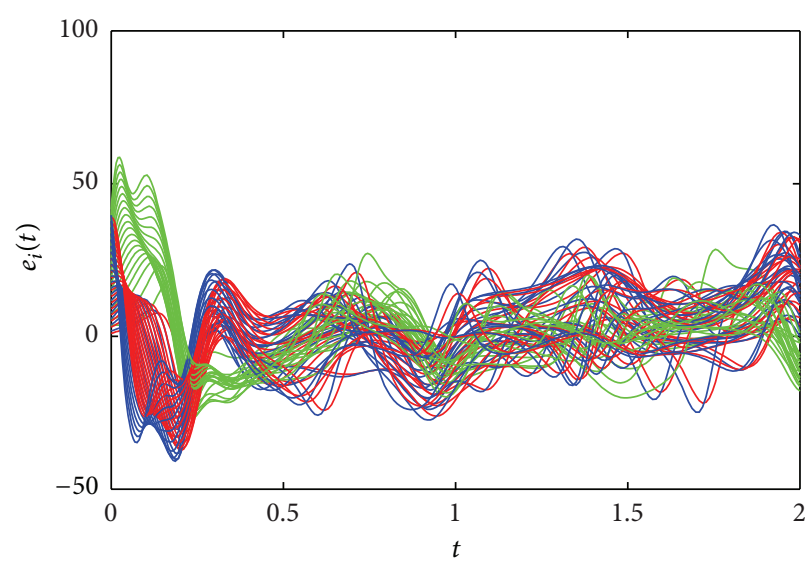

(b)

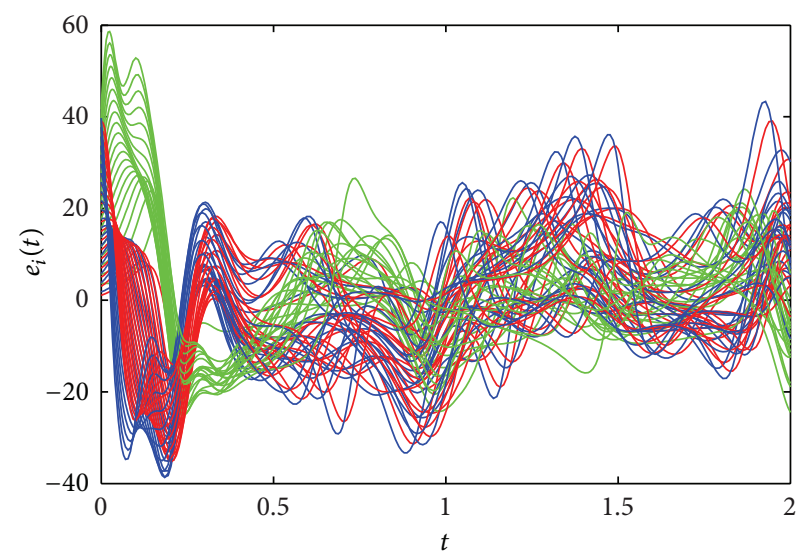

(d)

FIGURE 3: Separate synchronous variables $e_{i 1}, e_{i 2}, e_{i 3}(1 \leqslant i \leqslant 30)$ of different network models where $e_{i 1}$ are shown by the red line, $e_{i 2}$ are shown by the blue line, and $e_{i 3}$ are shown by the green line. $c=2 ; \tau=0.01$. The dynamics of nodes satisfy the Lü system. The nodes of the network cannot achieve synchronization without controllers. The network models of (a)-(d) are the same with Figures 1(a)-1(d) (color online).

and the controllers are designed as in (20)-(21). If the following condition holds:

$$
\lambda_{\text {max }}(A)+(1-v)\|A\|_{F}+L+c \lambda_{\max }\left(P_{0}\right)<d^{*},
$$

then the system (33) is synchronized, where $d^{*}$ is a sufficiently large positive constant to be determined.

\section{Numerical Simulation}

In this section, we use some examples to explain the influence of the proposed criteria, and we consider a network consisting of 30 similar nodes. The multilinks network with 2 properties can be described as follows:

$$
\begin{aligned}
\dot{x}_{i}(t)= & A_{i} x_{i}(t)+f\left(x_{i}(t)\right)+c \sum_{j=1}^{30} b_{(0) i j} \Gamma_{0} x_{j}(t) \\
& +c \sum_{j=1}^{30} b_{(1) i j} \Gamma_{1} x_{j}(t-\tau),
\end{aligned}
$$

where $1 \leqslant i \leqslant 30, B_{0}=\left(b_{(0) i j}\right)_{30 \times 30}$, and $B_{1}=\left(b_{(1) i j}\right)_{30 \times 30}$ are symmetrically diffusive coupling matrixes with $b_{(0) i j}$, $b_{(1) i j}=0$ or $1 . \Gamma_{0}=\Gamma_{1}=\operatorname{diag}(1,1,1), c=2, \tau=0.01$, $f\left(x_{i}(t)\right)=\left(0.6 \sin \left(x_{i 1}\right), 0.6 \sin \left(x_{i 2}\right), 0.6 \sin \left(x_{i 3}\right)\right)^{T}$. According to Assumption 3, we can know that $L=0.6$ :

$$
A_{i}=\left(\begin{array}{ccc}
-10+0.1 * \text { rand } & 0.1 * \text { rand } & 0.1 * \text { rand } \\
0.1 * \text { rand } & -10+0.1 * \text { rand } & 0.1 * \text { rand } \\
0.1 * \text { rand } & 0.1 * \text { rand } & -10+0.1 * \text { rand }
\end{array}\right),
$$

where the function of rand can produce a random number between 0 and 1 . According to the definition of similar nodes, we know $A_{i}, 1 \leqslant i \leqslant 30$ are matrices of the similar nodes. And

$$
A=\left(\begin{array}{ccc}
-10 & 0 & 0 \\
0 & -10 & 0 \\
0 & 0 & -10
\end{array}\right)
$$

is the matrix of the basic node. So

$$
\Delta A_{i}=\left(\begin{array}{lll}
0.1 * \text { rand } & 0.1 * \text { rand } & 0.1 * \text { rand } \\
0.1 * \text { rand } & 0.1 * \text { rand } & 0.1 * \text { rand } \\
0.1 * \text { rand } & 0.1 * \text { rand } & 0.1 * \text { rand }
\end{array}\right) .
$$

According to the precise calculation, $\lambda_{\max }(A)=-10$, $\lambda_{\max }\left(P_{0}\right)=0, \lambda_{\max }\left(P_{1}^{T} P_{1}\right)=18.5139$. Based on 


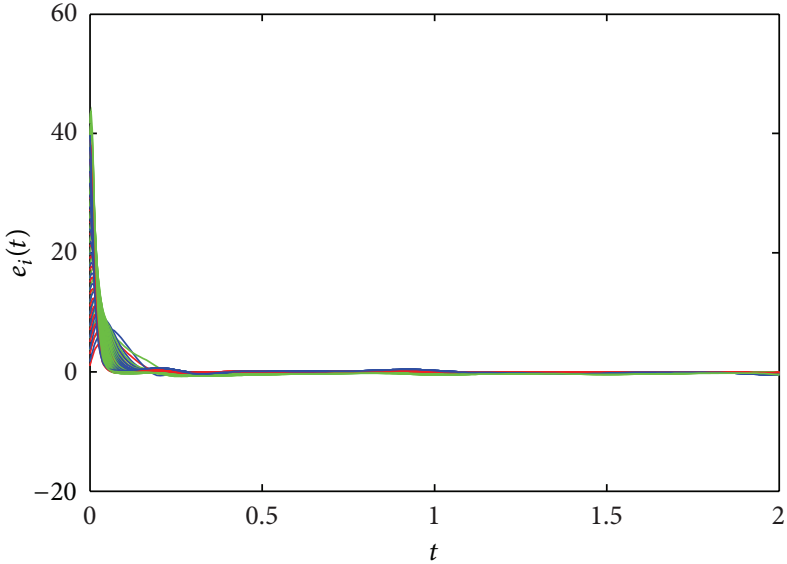

(a)

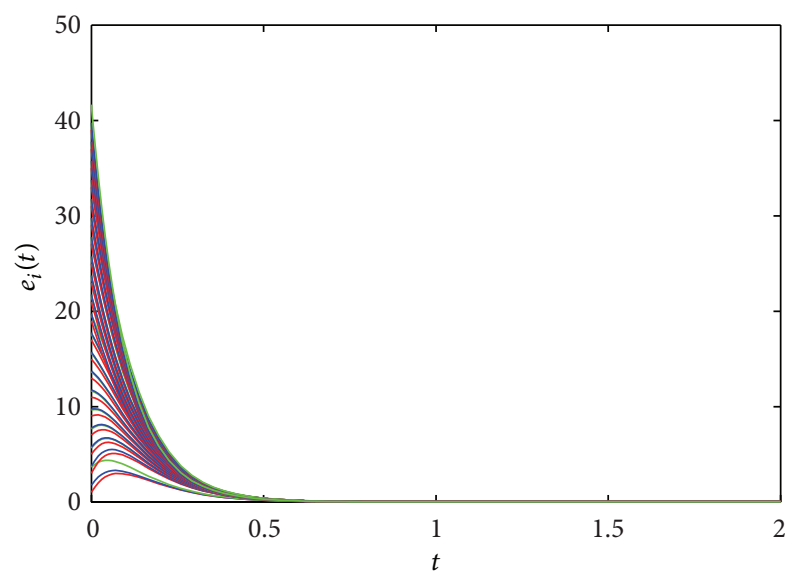

(c)

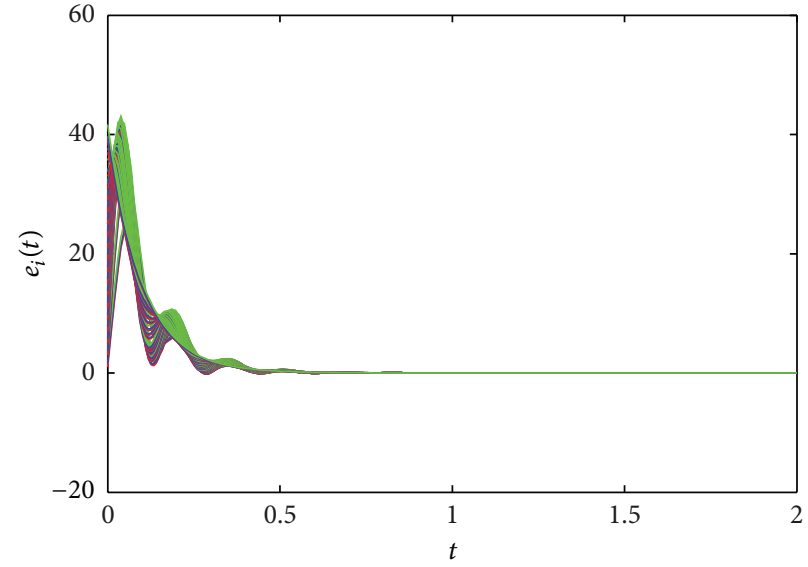

(b)

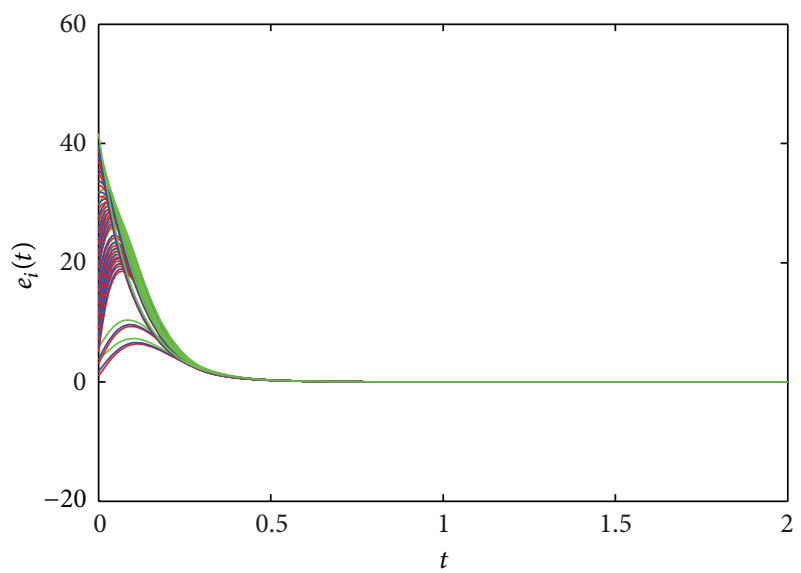

(d)

FIGURE 4: Separate synchronous variables $e_{i 1}, e_{i 2}, e_{i 3}(1 \leqslant i \leqslant 30)$ of different network models where $e_{i 1}$ are shown by the red line, $e_{i 2}$ are shown by the blue line, and $e_{i 3}$ are shown by the green line. $c=2 ; \tau=0.01$. The dynamics of nodes satisfy the Lü system. The nodes are controlled by the adaptive controllers (20) and (21). The network models of (a)-(d) are the same with Figures 1(a)-1(d) (color online).

the stability analysis, we get $\left(\lambda_{\max }(A)+L+c \lambda_{\max }\left(P_{0}\right)+\right.$ $\left.(c / 2) \sum_{l=1}^{l=m-1} \lambda_{\max }\left(P_{l} P_{l}^{T}\right)+(c / 2)(m-1)+\|A\|_{F}\right)\left(\|A\|_{F}\right)^{-1}=$ $(-100+0.6+18.5139+1+\sqrt{30000}) / \sqrt{30000}=0.5388$. According to (38), because $0<$ rand $<1$, the biggest changes are

$$
\Delta A_{i}=\left(\begin{array}{lll}
0.1 & 0.1 & 0.1 \\
0.1 & 0.1 & 0.1 \\
0.1 & 0.1 & 0.1
\end{array}\right) .
$$

Then we compute the $v=0.9983,0.5388<v<$ 1 , so the similar nodes can achieve synchronization which satisfies Theorem 6. From Figures 1(a)-1(d), we know that the similar nodes in the network achieved synchronization under different network models.

Furthermore, in order to verify Theorem 9, we choose the model (16) as the second example, where the Brownian motion satisfies $E \omega(t)=0, D \omega(t)=1$, and the parameters are the same with the first example. Figures $2(a)-2(d)$ plot the synchronous errors converge to 0 in finite time under different network models with noise, which reflects that similar nodes have a certain robustness. In our future work, we will consider the model (35) with Gaussian noise [25] or $1 / f$ noise [26], and the stochastic bounded model like [27] in the complex network with similar nodes will be studied.

Next, another example as the third one describes the controlled network using Lü systems and considers the network consisting of 30 nodes. The node dynamical system is $\dot{x}_{i}=\left(-36 x_{i 1}+36 x_{i 2} ; 20 x_{i 2}-x_{i 1} x_{i 3} ;-3 x_{i 3}+x_{i 1} x_{i 2}\right)$, for $i=1,2, \ldots, 30$. And $\Delta A_{i}$ are the same with the first example. Since Lü attractor is bounded, we suppose that all nodes are running in the given bounded region. There exists the constants $M_{1}=25, M_{2}=30, M_{3}=45$ satisfying $\left\|x_{i j}\right\|_{2},\left\|s_{j}\right\|_{2} \leqslant M_{j}$ for $1 \leqslant i \leqslant 30$ and $1 \leqslant j \leqslant 3$ [28]. Thus we have

$$
\left\|f\left(x_{i}\right)-f(s)\right\| \leq \sqrt{2 M_{1}^{2}+M_{2}^{2}+M_{3}^{2}}\left\|e_{i}\right\|_{2} \approx 64.6142\left\|e_{i}\right\|_{2},
$$




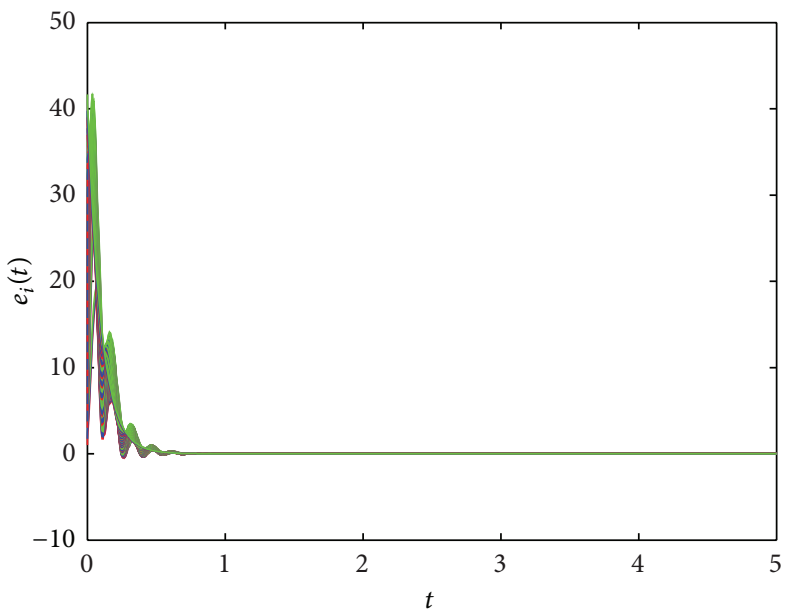

(a)

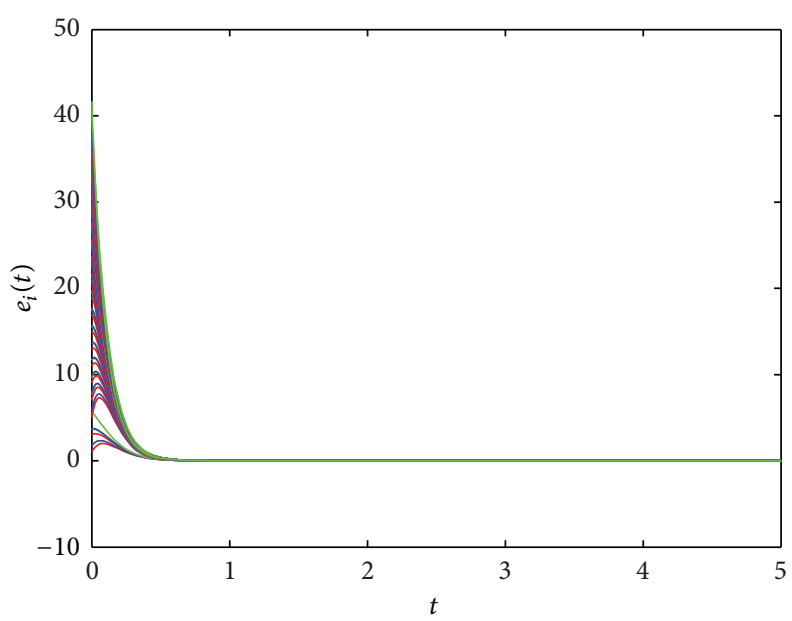

(c)

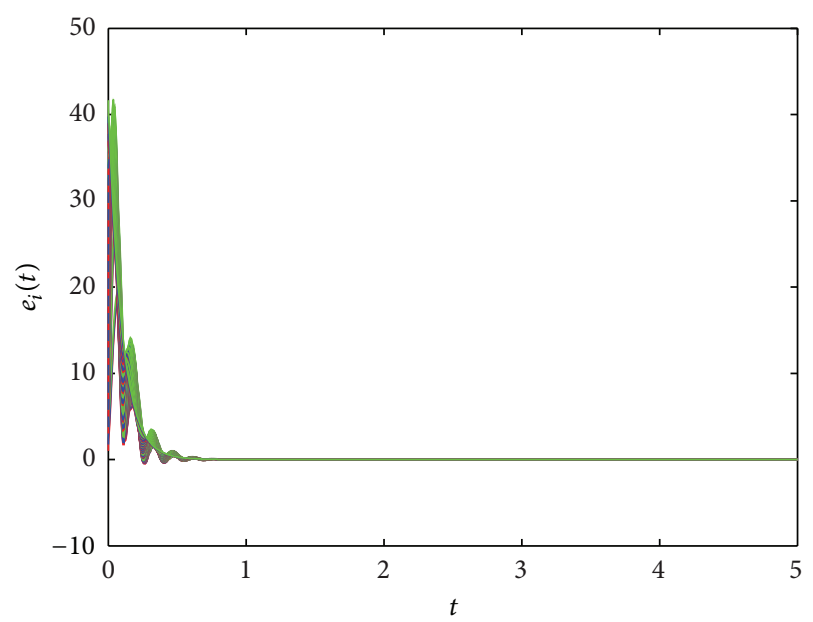

(b)

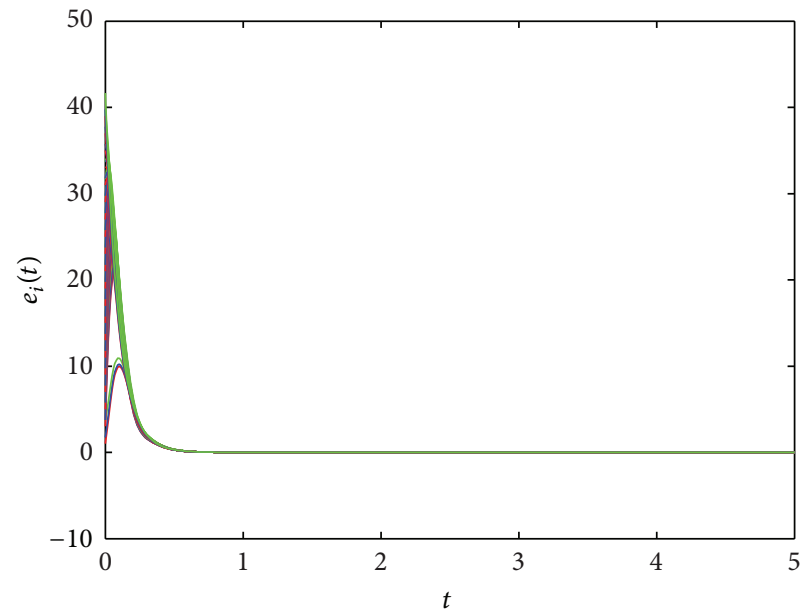

(d)

FIGURE 5: Separate synchronous variables $e_{i 1}, e_{i 2}, e_{i 3}(1 \leqslant i \leqslant 30)$ of different network models where $c=2, \tau=0.01$, and $\tau_{1}=0.01$. (a) $B_{0}, B_{1}$, and $B_{2}$ are small-world network models, the rewiring probability among nodes is $0.3,0.6$, and 0.7 . (b) $B_{0}, B_{1}$, and $B_{2}$ are scale-free network models, and their minimum degrees are 1,2, and 3. (c) $B_{0}, B_{1}$, and $B_{2}$ are random network models, and their connection probability among nodes is $0.1,0.3$, and 0.5 . (d) $B_{0}$ is a small-world network model, and the rewiring probability among nodes is 0.3 . $B_{1}$ is a scale-free network model, and the minimum degree is $2 . B_{2}$ is a random network model, and the connection probability among nodes is 0.1 . The dynamics of nodes satisfy the Lü system. The multilinks network can achieve synchronization under the adaptive controllers (color online).

then we can know that $L=64.6142$. And other parameters are the same with the first example. We have $\lambda_{\max }(A)=20$, and

$$
\begin{aligned}
& \left(\lambda_{\max }(A)+L+c \lambda_{\max }\left(P_{0}\right)\right. \\
& \left.+\frac{c}{2} \sum_{l=1}^{l=m-1} \lambda_{\max }\left(P_{l} P_{l}^{T}\right) \frac{c}{2}(m-1)+\|A\|_{F}\right)\left(\|A\|_{F}\right)^{-1} \\
& =\frac{20+64.6142+18.5139+1+\sqrt{3001}}{\sqrt{3001}}=2.9008 .
\end{aligned}
$$

It does not satisfy Theorem 6 . So the nodes cannot achieve synchronization without controllers. Simulation results are given in Figures 3(a)-3(d) which show the evolution process of 30 state variables in three dimensions. And it verified that the similar nodes cannot achieve synchronization without controllers.

According to the adaptive synchronization criteria, we add the adaptive controllers (20) and (21) to these similar nodes of the network. $k_{1}=k_{2}=k_{3}=1$. The curves of error vectors $e_{i 1}, e_{i 2}, e_{i 3}(i=1,2,3)$ are shown in Figures $4(\mathrm{a})-4(\mathrm{~d})$.

To be more persuadable, with the same calculation method, Figures 5(a)-5(d) plot the synchronous errors of networks with links owning 3 properties. Figures 5(a)$5(\mathrm{~d})$ have different network models, and $\tau_{1}=0.01$. This demonstrates that our theorem is not only applicable to multilinks network owning two links properties but also to real networks with multiple links. From Figures $1(\mathrm{a})-1$ (d) to Figures 5(a)-5(d), we attain that our theorems are feasible in different network models under different conditions. This 
result is more helpful to real networks not just to model networks. From the above simulation results, we can see that these similar nodes can achieve synchronization under the impacts of the adaptive controllers. In the future, we will consider the possible application of this paper to packet delay issue in computer communications.

\section{Conclusion}

In this paper, we present the definition of similar nodes and analyze their minimal similarity in the network for the first time. We find the threshold of the minimal similarity of the similar nodes if it is bigger than the threshold, then the similar nodes can achieve synchronization without controllers. Otherwise, we have to add some controllers in order to get synchronization. So some new adaptive synchronization criteria are proposed to realize the synchronization of multilinks networks with similar nodes. Finally, numerical simulations are provided to show the effectiveness and the correctness of the proposed criteria. The model and the principles designed in this paper are very useful to analyze and control the dynamical multilinks networks with similar nodes, such as heart cells networks and neural networks.

\section{Acknowledgments}

This paper is supported by the Foundation for the Author of National Excellent Doctoral Dissertation of PR China (Grant no. 200951), the National Natural Science Foundation of China (Grant nos. 61100204, 61070209, and 61121061), and the Asia Foresight Program under NSFC Grant (Grant no. 61161140320).

\section{References}

[1] S. H. Strogatz, "Exploring complex networks," Nature, vol. 410, no. 6825, pp. 268-276, 2001.

[2] A.-L. Barabási and R. Albert, "Emergence of scaling in random networks," Science, vol. 286, no. 5439, pp. 509-512, 1999.

[3] M. Chen, "Some simple synchronization criteria for complex dynamical networks," IEEE Transactions on Circuits and Systems II, vol. 53, no. 11, pp. 1185-1189, 2006.

[4] J. Lü and G. Chen, "A time-varying complex dynamical network model and its controlled synchronization criteria," IEEE Transactions on Automatic Control, vol. 50, no. 6, pp. 841-846, 2005.

[5] J. Cao, P. Li, and W. Wang, "Global synchronization in arrays of delayed neural networks with constant and delayed coupling," Physics Letters A, vol. 353, no. 4, pp. 318-325, 2006.

[6] J. Zhou, L. Xiang, and Z. Liu, "Global synchronization in general complex delayed dynamical networks and its applications," Physica A, vol. 385, no. 2, pp. 729-742, 2007.

[7] Y. Xu, W. Zhou, J. Fang, and H. Lu, "Structure identification and adaptive synchronization of uncertain general complex dynamical networks," Physics Letters A, vol. 374, no. 2, pp. 272278, 2009.

[8] H. Peng, N. Wei, L. Li, W. Xie, and Y. Yang, "Models and synchronization of time-delayed complex dynamical networks with multi-links based on adaptive control," Physics Letters A, vol. 374, no. 23, pp. 2335-2339, 2010.
[9] H. Du, P. Shi, and N. Lü, "Function projective synchronization in complex dynamical networks with time delay via hybrid feedback control," Nonlinear Analysis: Real World Applications, vol. 14, no. 2, pp. 1182-1190, 2013.

[10] J. Lu, D. W. C. Ho, and J. Cao, "A unified synchronization criterion for impulsive dynamical networks," Automatica, vol. 46, no. 7, pp. 1215-1221, 2010.

[11] Z.-H. Guan, Z.-W. Liu, G. Feng, and Y.-W. Wang, "Synchronization of complex dynamical networks with time-varying delays via impulsive distributed control," IEEE Transactions on Circuits and Systems I, vol. 57, no. 8, pp. 2182-2195, 2010.

[12] J. Lu, D. W. C. Ho, J. Cao, and J. Kurths, "Exponential synchronization of linearly coupled neural networks with impulsive disturbances," IEEE Transactions on Neural Networks, vol. 22, no. 2, pp. 329-335, 2011.

[13] Y. Xu, W. Zhou, J. Fang, and W. Sun, "Adaptive lag synchronization and parameters adaptive lag identification of chaotic systems," Physics Letters A, vol. 374, no. 34, pp. 3441-3446, 2010.

[14] Y. Xu, W. Zhou, J. Fang, and W. Sun, "Adaptive synchronization of the complex dynamical network with non-derivative and derivative coupling," Physics Letters A, vol. 374, no. 15-16, pp. 1673-1677, 2010.

[15] A. Das and F. L. Lewis, "Distributed adaptive control for synchronization of unknown nonlinear networked systems," Automatica, vol. 46, no. 12, pp. 2014-2021, 2010.

[16] X. F. Wang and G. Chen, "Pinning control of scale-free dynamical networks," Physica A, vol. 310, no. 3-4, pp. 521-531, 2002.

[17] X. Li, X. Wang, and G. Chen, "Pinning a complex dynamical network to its equilibrium," IEEE Transactions on Circuits and Systems I, vol. 51, no. 10, pp. 2074-2087, 2004.

[18] F. Sorrentino, M. Bernardo, F. Garofalo, and G. Chen, "Controllability of complex networks via pinning," Physical Review E, vol. 75, no. 4, Article ID 046103, 6 pages, 2007.

[19] Q. Hu, H. Peng, Y. Wang, Z. Hu, and Y. Yang, "Pinning adaptive synchronization of complex dynamical network with multilinks," Nonlinear Dynamics, vol. 69, no. 4, pp. 1813-1824, 2012.

[20] J. Zhou, J.-A. Lu, and J. Lü, "Pinning adaptive synchronization of a general complex dynamical network," Automatica, vol. 44, no. 4, pp. 996-1003, 2008.

[21] W. Yu, G. Chen, and J. Lü, "On pinning synchronization of complex dynamical networks," Automatica, vol. 45, no. 2, pp. 429-435, 2009.

[22] Y. Wang, Y. Fan, Q. Wang, and Y. Zhang, "Stabilization and synchronization of complex dynamical networks with different dynamics of nodes via decentralized controllers," IEEE Transactions on Circuits and Systems I, vol. 59, no. 8, pp. 1786-1795, 2012.

[23] Q. Song, J. Cao, and F. Liu, "Synchronization of complex dynamical networks with nonidentical nodes," Physics Letters A, vol. 374, no. 4, pp. 544-551, 2010.

[24] S. Cai, Q. He, J. Hao, and Z. Liu, "Exponential synchronization of complex networks with nonidentical time-delayed dynamical nodes," Physics Letters A, vol. 374, no. 25, pp. 2539-2550, 2010.

[25] M. Li and W. Zhao, "Quantitatively investigating locally weak stationarity of modified multifractional Gaussian noise," Physica A, vol. 391, no. 24, pp. 6268-6278, 2012.

[26] M. Li and W. Zhao, "On $1 / f$ noise," Mathematical Problems in Engineering, vol. 2012, Article ID 673648, 23 pages, 2012.

[27] M. Li and W. Zhao, "Representation of a stochastic traffic bound," IEEE Transactions on Parallel and Distributed Systems, vol. 21, no. 9, pp. 1368-1372, 2010. 
[28] D. Li, J.-A. Lu, X. Wu, and G. Chen, "Estimating the ultimate bound and positively invariant set for the Lorenz system and a unified chaotic system," Journal of Mathematical Analysis and Applications, vol. 323, no. 2, pp. 844-853, 2006. 


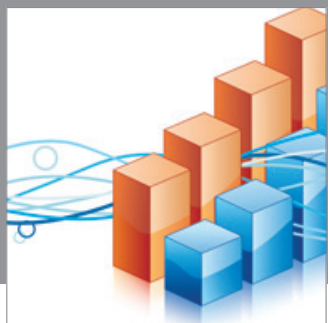

Advances in

Operations Research

mansans

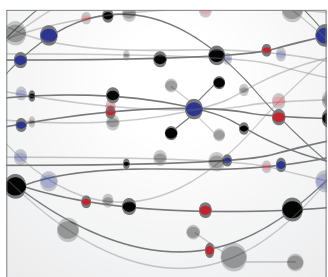

The Scientific World Journal
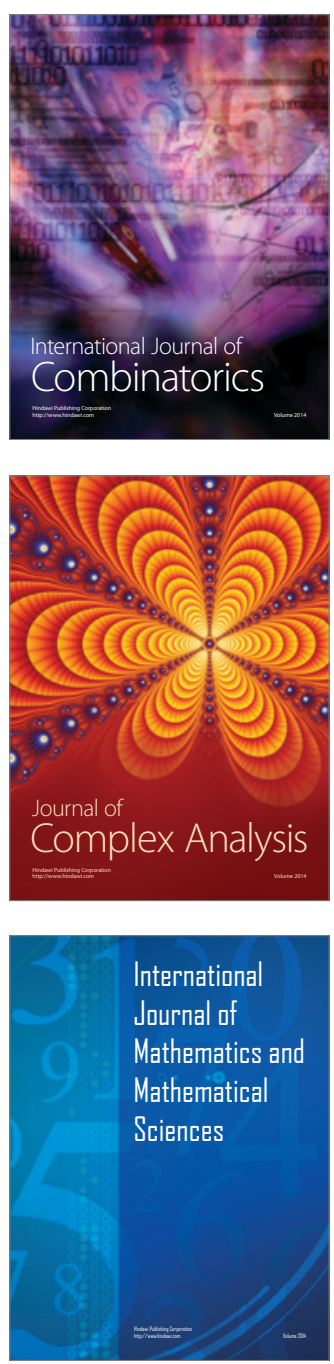
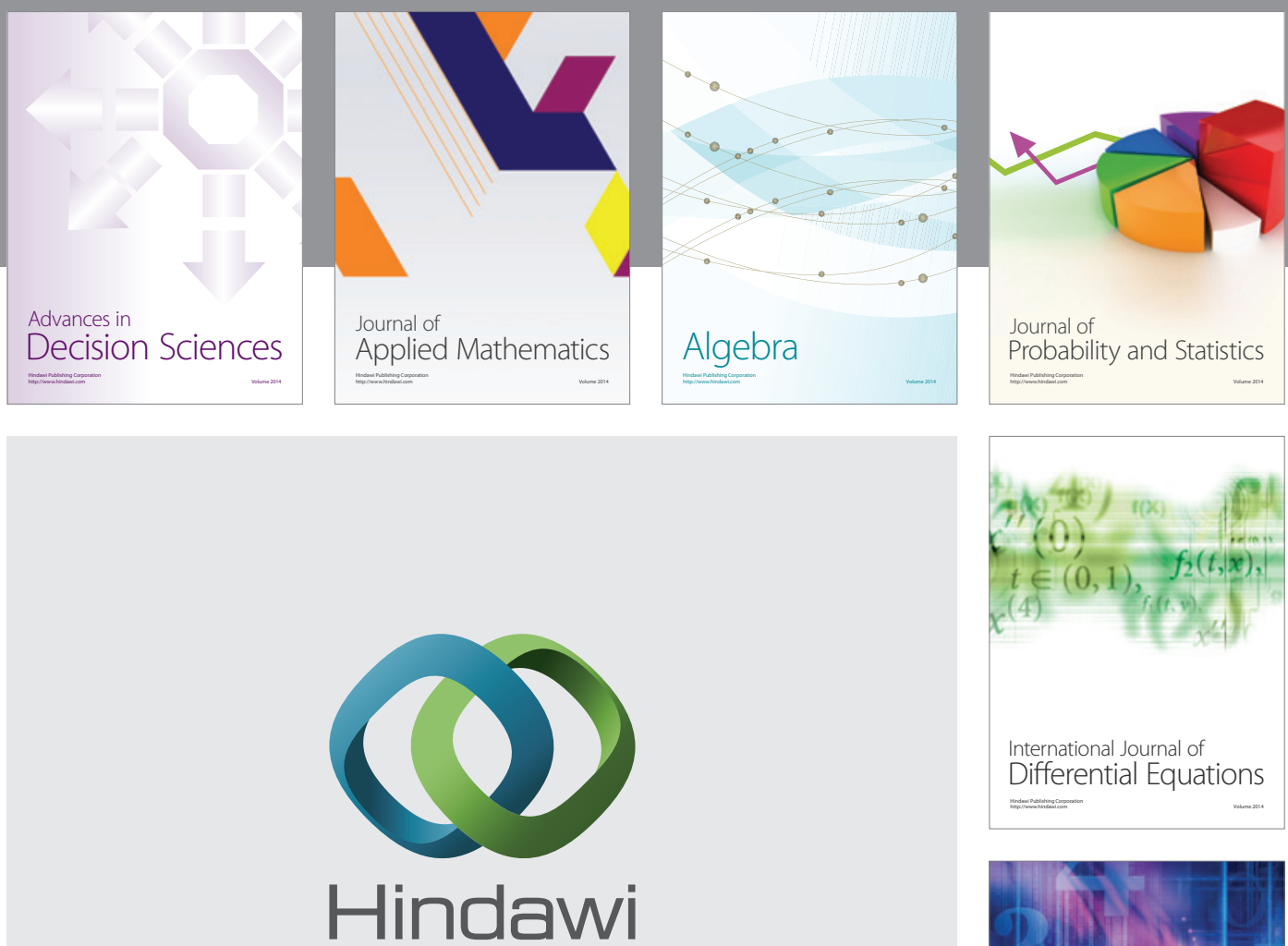

Submit your manuscripts at http://www.hindawi.com
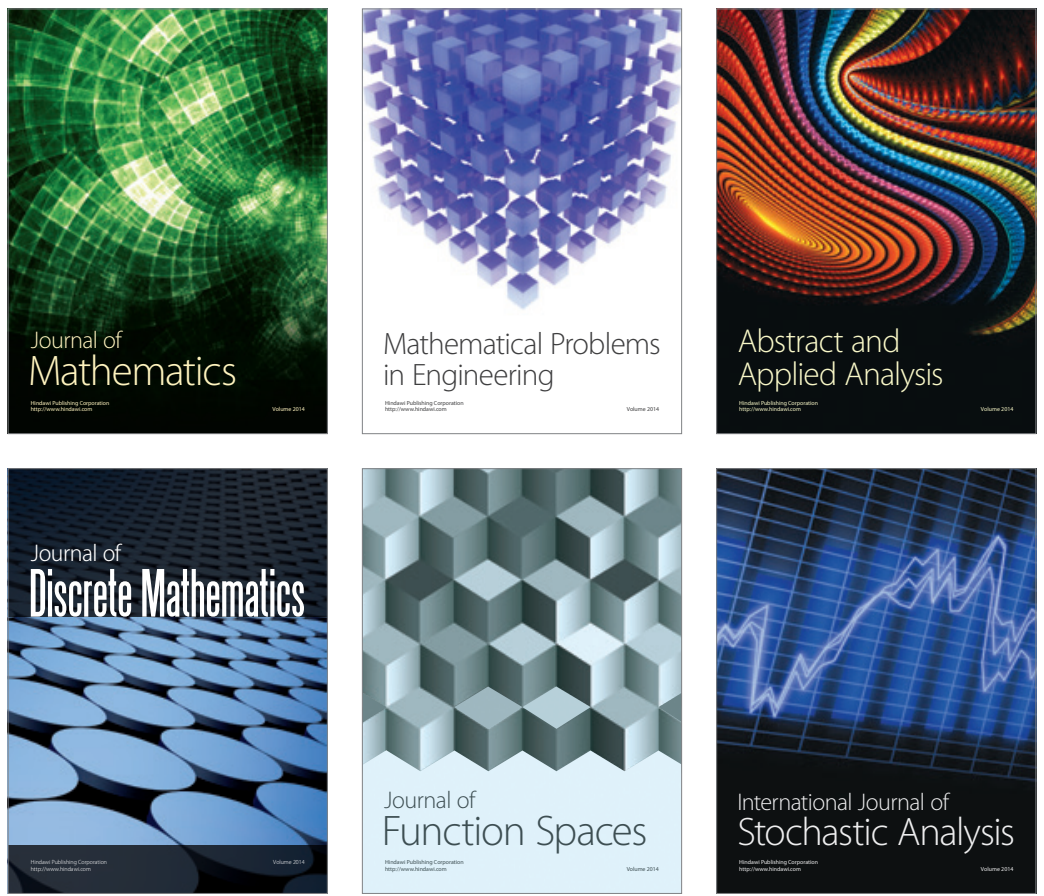

Journal of

Function Spaces

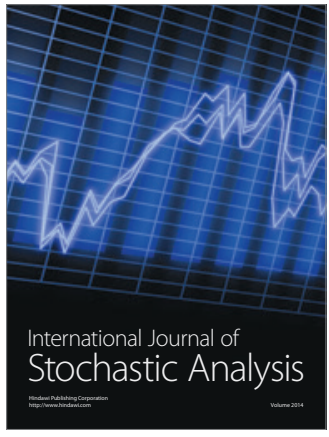

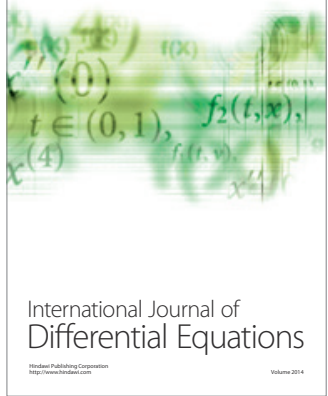
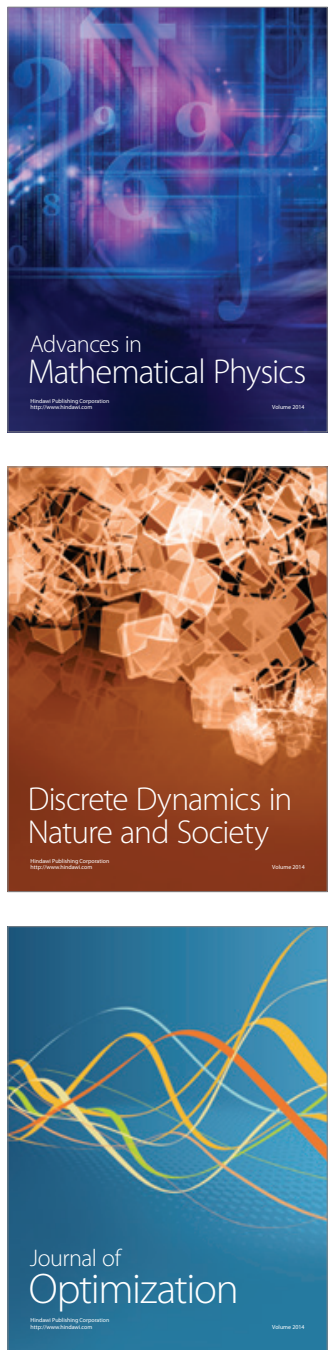